\title{
Resistance of multilayers with long length scale interfacial roughness
}

Cite as: Journal of Applied Physics 93, 7930 (2003); https://doi.org/10.1063/1.1555799

Published Online: 09 May 2003

Jason Alicea, and Selman Hershfield

\section{ARTICLES YOU MAY BE INTERESTED IN}

Correlation between spin polarization and magnetic moment in ferromagnetic alloys

Journal of Applied Physics 86, 562 (1999); https://doi.org/10.1063/1.370766 


\title{
Resistance of multilayers with long length scale interfacial roughness
}

\author{
Jason Alicea ${ }^{\text {a) }}$ and Selman Hershfield ${ }^{\text {b) }}$ \\ Department of Physics and National High Magnetic Field Laboratory, University of Florida, Gainesville, \\ Florida 32611-8440
}

(Presented on 14 November 2002)

\begin{abstract}
The resistance of multilayers with interfacial roughness on a length scale large compared to the layer spacing is obtained using the Boltzmann equation. Both the current-perpendicular-to-plane (CPP) and current-in-plane (CIP) geometries are considered in the limits where the mean-free paths are short and long compared to the atomic spacing. In the short mean-free path limit, the resistance decreases in the CPP geometry and increases in the CIP geometry. In the long mean-free path limit, the resistance increases in both configurations due to enhanced surface scattering. The giant magnetoresistance can either be enhanced or reduced by roughness depending on the sample parameters. Estimates of the short and long mean-free path effects in $\mathrm{Fe} / \mathrm{Cr}$ multilayers are obtained using experimentally determined parameters. (C) 2003 American Institute of Physics.
\end{abstract}

[DOI: $10.1063 / 1.1555799]$

\section{INTRODUCTION}

One of the controversial questions about the giant magnetoresistance (GMR) is how its magnitude is effected by the presence of long length scale interface disorder, i.e., interfacial roughness on a length scale which is large compared to the atomic spacing. Several experiments have studied the effects of this type of disorder in both the geometries where the current flows perpendicular to the layers (CPP geometry $)^{1-4}$ and in the plane of the layers (CIP geometry) ${ }^{5-10}$ Although current theories are consistent with the CIP experiments, ${ }^{11,12}$ there are several unexplained experiments in the CPP geometry which yield apparently contradictory results.

Chiang et al. ${ }^{2}$ studied the role of interface disorder in $\mathrm{Co} / \mathrm{Ag}$ multilayers and found that the CPP magnetoresistance was reduced by increasing interfacial roughness while the CIP magnetoresistance was enhanced. In a set of experiments on Fe/Cr multilayers, Cyrille et al. ${ }^{1}$ found that both the CPP and CIP magnetoresistances were enhanced by interfacial roughness. In the CPP case, the enhancement of the magnetoresistance was due to the zero-field resistivity, $\rho_{A P}$, increasing with roughness while the high-field resistivity, $\rho_{P}$, remained roughly constant. Still, more recent experiments by Zambano et al. ${ }^{4}$ see no change in the CPP resistivities or the magnetoresistance of $\mathrm{Fe} / \mathrm{Cr}$ multilayers with increasing roughness.

The effect of interfacial roughness on the CPP GMR is therefore unclear, with some work pointing to an enhancement of the GMR, some a reduction, and others no change at all. In this article, we examine theoretically the effects of long length scale interface disorder in both the CIP and CPP geometries using the Boltzmann equation. ${ }^{13,14}$

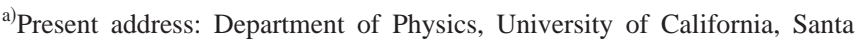
Barbara, CA 93117; electronic mail: alicea@physics.ucsb.edu

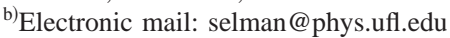

\section{THEORETICAL FRAMEWORK}

The multilayers we studied are shown in Fig. 1. The layers are separated by sinusoidal interfaces with amplitude $A$ and period $\xi$, and within each layer the relaxation time, $\tau_{i}$, is constant. For numerical purposes, our calculations are performed in two dimensions, although we nonetheless expect the results to remain qualitatively the same when generalized to three dimensions.

The Boltzmann equation we used represents elastic $s$-wave scattering within a current-conserving right-hand side:

$$
\mathbf{v} \cdot \nabla_{r} f-e \mathbf{E} \cdot \nabla_{p} f=-\left(\frac{f-\bar{f}}{\tau}\right),
$$

where $f=f(\mathbf{r}, \mathbf{p})$ is the distribution function, $\bar{f}=\bar{f}(\mathbf{r},|\mathbf{p}|)$ is

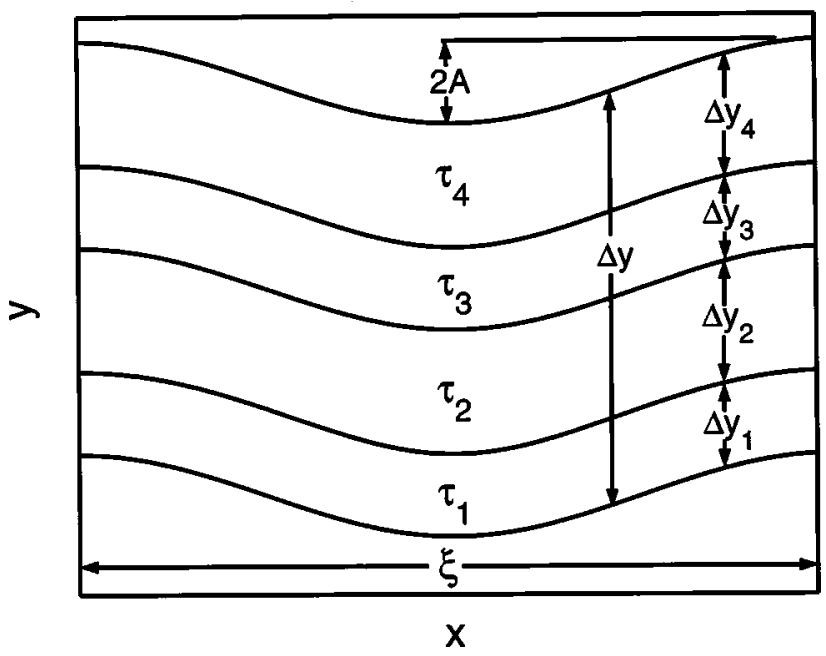

FIG. 1. Geometry of the multilayers studied. The interfaces are modeled as sine waves with amplitude $A$ and period $\xi$. Layer $i$ has a thickness $\Delta y_{i}$ and uniform relaxation time $\tau_{i}$, and the total thickness of the sample is $\Delta y$. The current flows in the $y$ direction in the CPP configuration and the $x$ direction in the CIP configuration. 
the spherical average of $f$ in momentum space, and $\tau=\tau(\mathbf{r})$ is the relaxation time. For flat interfaces, the linear response solution to this Boltzmann equation can be obtained analytically. Such a solution is not possible when long length scale interfacial disorder is present; however, there are two limiting cases for which one can obtain an essentially exact linear response solution for Eq. (1): The short and long mean-free path limits. ${ }^{15}$ These correspond to the cases where the electronic mean-free paths are much smaller and much larger than the layer thicknesses, respectively. In the short meanfree path limit, the layers behave as macroscopic pieces of metal and can essentially be regarded as resistors in series (CPP geometry) or parallel (CIP geometry). Here, the resistance of the multilayer is governed by classical transport equations, and the Boltzmann equation reduces to a Laplace equation for the electrochemical potential within each layer. In the long mean-free path limit, variations across the sample are smeared out, and the multilayer behaves as a homogeneous, isotropic metal. In this case, the gradient of the electrochemical potential can be approximated by its average value, and the Boltzmann equation may be integrated directly. ${ }^{15}$

In order to compare with experiments, it is crucial to include surface scattering in these calculations. In the short mean-free path limit, we model surface scattering by inserting a thin layer of a higher-resistivity material between the bulk layers. In the long mean-free path limit, surface scattering is included on the right-hand side of Eq. (1) in addition to the bulk scattering term, $1 / \tau_{b}$, by writing

$$
\frac{1}{\tau(\mathbf{r})}=\frac{1}{\tau_{b}(\mathbf{r})}+\Gamma \sum_{i} \int d \ell \delta^{2}\left(\mathbf{r}-\mathbf{R}_{i}(\ell)\right),
$$

where the integration runs along the $i$ th interface, $\mathbf{R}_{i}(\ell)$ is the position of the $i$ th boundary, and $\Gamma$ is a parameter characterizing the surface scattering rate. Equation (2) can be derived by modeling the interfaces as infinitesimal layers of a higher-resistivity material.

\section{RESULTS}

The solution of the Boltzmann equation in the short mean-free path limit shows that the effective CPP conductivity increases with roughness while the CIP conductivity decreases. These effects can be understood in terms of the current. In the CPP geometry, roughness allows the current to traverse a less-resistive, nonlinear path through the sample that reduces the distance traveled through the low conductivity layers. In the CIP geometry, where the layers behave essentially as parallel resistors, most of the current short circuits through the low resistance layers. Due to interfacial roughness, some of the current flowing through these layers will be forced to impinge on more resistive layers near the interfaces. This reduces the short-circuit effect of the high conductivity layers, leading to a decrease in the effective conductivity.

Quantitatively, in both geometries the fractional change in the conductivity due to roughness is proportional to $(A / \xi)^{2}$, where the proportionality constant has a different magnitude in the CPP and CIP configurations and depends on the layer conductivities and sample geometry. Estimates for these proportionality constants can be obtained by approximating the interfaces as grooved rather than sinusoidal: ${ }^{12,15}$

$$
\frac{\delta \sigma_{\mathrm{CIP} / \mathrm{CPP}}}{\sigma_{\mathrm{CIP} / \mathrm{CPP}}} \approx \pm 16\left(\frac{\sigma_{\mathrm{CPP}}}{\sigma_{\mathrm{CIP}}}-1\right)\left(\frac{A}{\xi}\right)^{2},
$$

where $\sigma_{\mathrm{CPP}}$ and $\sigma_{\mathrm{CIP}}$ are the CPP and CIP conductivities for flat interfaces. Equation (3) only roughly approximates the exact proportionality constants computed numerically, but it is useful for comparing to experiments. ${ }^{15}$

In the long mean-free path limit, we find that the conductivity decreases with roughness due entirely to enhanced surface scattering. When surface scattering is ignored, the conductivity is independent of roughness. To find how the surface scattering is effected quantitatively by roughness, we extract the interface conductivity, $\sigma^{*}$, by treating the bulk and interface resistances as resistors in series. To a good approximation, the fractional decrease in the interface conductivity is equal to the fractional change in the interface length resulting from roughness, independent of the model parameters. For the sinusoidal boundaries we consider, the fractional change in the interface conductivity is

$$
\frac{\delta \sigma^{*}}{\sigma^{*}} \approx-\pi^{2}\left(\frac{A}{\xi}\right)^{2} .
$$

Note that in the long mean-free path limit the conductivity is isotropic, and the CPP and CIP conductivities are the same.

To get the GMR, the difference of the resistivities in the antiparallel $\left(\rho_{A P}\right)$ and parallel $\left(\rho_{P}\right)$ configurations is computed: $\operatorname{GMR}=\left(\rho_{A P}-\rho_{P}\right) / \rho_{P}$. In the short mean-free path limit, when the resistance of each channel is computed numerically, we find that roughness can either enhance or reduce the CPP GMR depending on the sample parameters, while in the CIP geometry, roughness induces a positive GMR that vanishes when the interfaces are flat. In the long mean-free path limit, we also find that the GMR can be either enhanced or reduced by roughness depending on the parameters.

In an actual experiment, the effects in the two limits discussed herein will both be present to some extent, as the mean-free paths will be in neither limit, but somewhere in between. To see if we can account for the measurements of Cyrille et al. ${ }^{1}$ and Zambano et al. ${ }^{4}$ we now estimate the size of these two effects by computing the high-field and zerofield resistivities in $\mathrm{Fe}(3 \mathrm{~nm}) / \mathrm{Cr}(1.2 \mathrm{~nm})$ multilayers as a function of the number of bilayers $N$ using experimentally determined parameters. The roughness parameters $A$ and $\xi$ in our model were determined experimentally by Cyrille et al. ${ }^{1,3}$ They found that $A$ increased within the multilayer while $\xi$ remained constant at $10 \mathrm{~nm}$. Thus, the interfaces in their samples become more disordered as the sample size is increased. We used for our calculations the average value of $(A / \xi)^{2}$ across the multilayer. The bulk and interface resistances have been determined by Zambano et al. ${ }^{4}$ We used their measurements for the estimates because the resistances in the two sets of experiments are roughly the same magnitude even though the trends are different. 


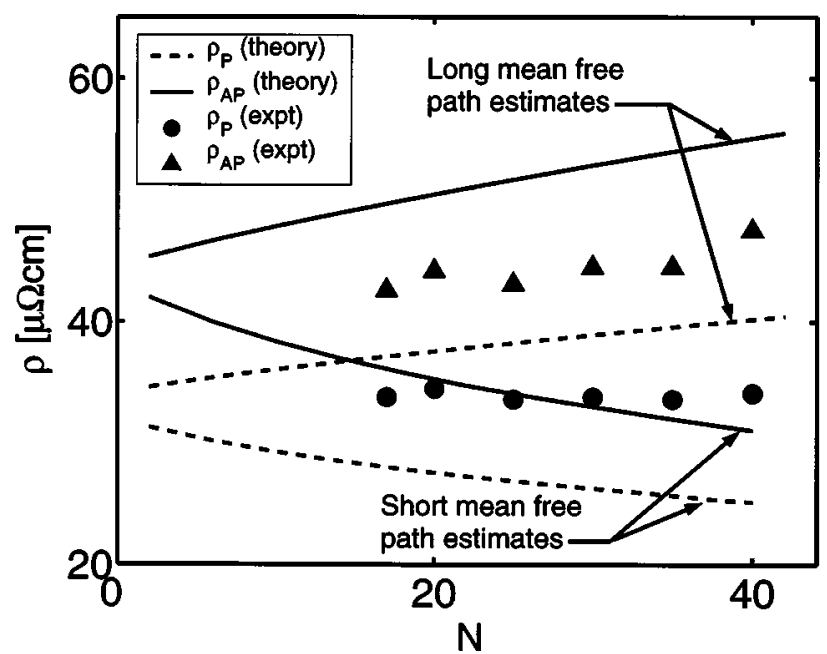

FIG. 2. Long and short mean-free path estimates of the parallel and antiparallel resistivities for $\mathrm{Fe}(3 \mathrm{~nm}) / \mathrm{Cr}(1.2 \mathrm{~nm})$ multilayers as a function of the number of bilayers $N$. For comparison, measurements [obtained by Cyrille et al. (Ref. 1)] are also shown. Since the changes in the resistivities predicted in the two limits are opposite in sign, there can be significant cancelation of the two effects in samples with intermediate mean-free paths. Thus, depending on the mean-free paths, these results may account for either the trends [seen by Cyrille et al. (Ref. 1)] shown in the figure or the negligible changes in the resistivities [observed by Zambano et al. (Ref. 4)] in the same type of samples.

The results of these estimates in the CPP geometry, along with the corresponding measurements obtained by Cyrille et al., ${ }^{1}$ are shown in Fig. 2. The resistivity measurements obtained by Zambano et al. ${ }^{4}$ are similar in magnitude but do not depend on $N$. Because the changes in the resistivities predicted in the long and short mean-free path limits are opposite in sign and of roughly the same magnitude, these estimates show that the resistivities can either increase, decrease, or remain constant with roughness depending on which effect is dominant. If the long mean-free path effect is more important, one would expect trends similar to those measured by Cyrille et al. ${ }^{1}$ in the intermediate mean-free path case because $\rho_{A P}$ increases more than $\rho_{P}$ in the long mean free path limit. If the long and short mean-free path effects cancel, however, one would expect to see the negligible changes in the resistivities observed by Zambano et $\mathrm{al}^{4}{ }^{4}$ Thus, the effects described here are of the correct size to explain what is seen experimentally, but a theory which accounts for an arbitrary mean-free path is needed in order to make a detailed quantitative comparison to experiment.

\section{ACKNOWLEDGMENTS}

The authors would like to thank Tat-Sang Choy, Jack Bass, and Ivan Schuller for helpful discussions. This research was supported by the DOD/AFOSR Grant No. F49620-961-0026, the Center for Condensed Matter Sciences, the University Scholars Program at the University of Florida, and the National Science Foundation through the U.F. Physics REU Program.

${ }^{1}$ M. C. Cyrille, S. Kim, M. E. Gomez, J. Santamaria, K. M. Krishnan, and I. K. Schuller, Phys. Rev. B 62, 3361 (2000)

${ }^{2}$ W. C. Chiang, W. P. Pratt, M. Herrold, and D. V. Baxter, Phys. Rev. B 58, 5602 (1998)

${ }^{3}$ J. Santamaria, M.-E. Gomez, M.-C. Cyrille, C. Leighton, K. M. Krishnan, and I. K. Schuller, Phys. Rev. B 65, 12412 (2002).

${ }^{4}$ A. Zambano, K. Eid, R. Loloee, W. P. Pratt, and J. Bass, J. Magn. Magn. Mater. 253, 51 (2002).

${ }^{5}$ E. E. Fullerton, D. M. Kelly, J. Guimpel, I. K. Schuller, and Y. Bruynseraede, Phys. Rev. Lett. 68, 859 (1992).

${ }^{6}$ J. M. Colino, I. K. Shuller, V. Korenivski, and K. V. Rao, Phys. Rev. B 54, 13030 (1996).

${ }^{7}$ M. Velez and I. K. Schuller, J. Magn. Magn. Mater. 184, 275 (1998).

${ }^{8}$ R. Schad, J. Barnas, P. Beliën, G. Verbanck, C. D. Potter, H. Fischer, S. Lefebvre, M. Bessiere, V. V. Moshchalkov, and Y. Bruynseraede, J. Magn. Magn. Mater. 156, 339 (1996).

${ }^{9}$ R. Schad, P. Beliën, G. Verbanck, C. D. Potter, H. Fischer, S. Lefebvre, M. Bessiere, V. V. Moshchalkov, and Y. Bruynseraede, Phys. Rev. B 57, 13692 (1998).

${ }^{10}$ R. Schad, P. Beliën, G. Verbanck, K. Temst, V. V. Moshchalkov, Y. Bruynseraede, D. Bahr, J. Falta, J. Dekoster, and G. Gangouche, Europhys. Lett. 44, 379 (1998).

${ }^{11}$ J. Barnas and Y. Bruynseraede, Phys. Rev. B 53, 5449 (1996).

${ }^{12}$ P. M. Levy, S. Zhang, T. Ono, and T. Shinjo, Phys. Rev. B 52, 16049 (1995).

${ }^{13}$ R. E. Camley and J. Barnas, Phys. Rev. Lett. 63, 664 (1989).

${ }^{14}$ T. Valet and A. Fert, Phys. Rev. B 48, 7099 (1993).

${ }^{15} \mathrm{~J}$. Alicea and S. Hershfield, cond-mat/0208251 (2002). 